\title{
Recent developments in hydrodynamics and collectivity in small systems
}

\author{
Harri Niemi* \\ Institut für Theoretische Physik, Johann Wolfgang Goethe-Universität, Max-von-Laue-Str. 1, \\ D-60438 Frankfurt am Main, Germany \\ E-mail: niemiath.physik.uni-frankfurt.de
}

\begin{abstract}
A short overview of the current status of describing spacetime evolution of the hot matter created in relativistic nuclear collisions with fluid dynamics and of determining the transport coefficients of strongly interacting matter is given. The comparisons with the experimental data and the fluid dynamical models suggest that a small droplet of fluid with a very low viscosity is created in these collisions. However, there are still challenges in describing these events, especially in constraining the temperature dependence of the transport coefficients, and in understanding the applicability limits of the fluid dynamical approaches.
\end{abstract}

The European Physical Society Conference on High Energy Physics 22-29 July 2015

Vienna, Austria

${ }^{*}$ Speaker. 
One of the fundamental questions in high-energy physics is what are the properties of strongly interacting matter at high temperature and density. In sufficiently high temperature or density one expects a transition from hadronic matter to a deconfined state, quark-gluon plasma (QGP), where the degrees of freedom are quarks and gluons, instead of their bound states hadrons.

Experimentally the properties of such systems can be studied in relativistic heavy ion collisions, where it is believed that small amount of nearly thermalized strongly interacting matter is created. Currently, there are two major colliders: RHIC at BNL and the LHC at CERN that are providing a wealth of experimental data, giving detailed constraints on the properties of QGP. In the future, the FAIR experiment will give more information about the behavior of the matter at high baryon density.

A natural theoretical framework to investigate the properties of nearly thermalized matter is that of fluid dynamics, which is a limit, where the dynamics of the system is completely determined by a few macroscopic properties like viscosity (transport coefficients), and equation of state. Fluid dynamics is also a convenient framework to use in constraining the transport properties, as the transport coefficients and equation of state are direct inputs to the models. However, applying fluid dynamics also requires a separation between the microscopic and macroscopic time and space scales, usually characterized by their ratio, the Knudsen number. For system as small as those created in the heavy ion collisions it is not at all trivial that the Knudsen number is sufficiently small. However, the system is also strongly interacting, and the predictions of fluid dynamical models are in an extremely good agreement with the experimental data, suggesting that indeed a small droplet of fluid is created in these collisions. For recent reviews, see Refs. [1]

\section{Fluid dynamics}

Basic equations governing the fluid dynamical evolution are the conservation laws $\partial_{\mu} T^{\mu v}=0$ and $\partial_{\mu} N_{i}^{\mu}=0$, where $T^{\mu \nu}$ is the energy-momentum tensor and $N_{i}^{\mu}$ are the possible additional conserved currents (charge, baryon number, etc.) In general $T^{\mu v}$ can be decomposed w.r.t. fluid 4-velocity $u^{\mu}$, defined for example in the Landau frame $e_{0} u^{\mu}=T^{\mu v} u_{v}$, as $T^{\mu v}=e_{0} u^{\mu} u^{v}-P \Delta^{\mu v}+$ $\pi^{\mu v}$, where $e_{0}$ is the local energy density $P=P_{0}+\Pi$ is the isotropic pressure (sum of equilibrium pressure $P_{0}$ and bulk viscous pressure $\Pi$ ), and $\pi^{\mu v}$ is the shear-stress tensor.

The conservation laws are obviously always fulfilled, but they alone do not provide enough constraints to determine the evolution. The fluid dynamical limit is essentially realized by providing the closure relations that give the evolution of the dissipative quantities like $\pi^{\mu \nu}$. For example, in the simplest Navier-Stokes (NS) approximation the shear-stress tensor and bulk viscous pressure are directly proportional to the gradients of velocity, i.e. $\pi^{\mu v}=2 \eta\left(T,\left\{\mu_{i}\right\}\right) \nabla^{\langle\mu} u^{v\rangle}$ and $\Pi=-\zeta\left(T,\left\{\mu_{i}\right\}\right) \nabla_{\mu} u^{\mu}$. The microscopic properties of the matter then determine the transport coefficients $\eta\left(T,\left\{\mu_{i}\right\}\right)$ and $\zeta\left(T,\left\{\mu_{i}\right\}\right)$, which in general depend on the temperature $T$ and the chemical potentials $\left\{\mu_{i}\right\}$ associated with the conserved charges.

The problem with the relativistic NS theory is that even the hydrostatic equilibrium is unstable under small perturbations [2,3]. This follows essentially from the fact that in the NS theory the signal propagation speeds are not bounded by the speed of light, and consequently the theory is not causal. This makes the relativistic NS theory unusable for the full dynamical description of the system, and therefore it is necessary to use approximations going beyond the simple NS theory. 
The most of the analyses of heavy ion collisions are based on the Israel-Stewart type of theories [4], where part of the microscopic transient dynamics is taken into account, i.e. the dissipative quantities relax towards the NS values given by the gradients and the viscosities, within a characteristic relaxation time $\tau$ and not instantaneously like in the NS theory. For example, by neglecting the bulk viscosity and heat conductivity, the evolution equations of the shear-stress tensor can be written as

$$
\tau_{\pi} \frac{d}{d \tau} \pi^{\langle\mu v\rangle}+\pi^{\mu v}=2 \eta \nabla^{\langle\mu} u^{v\rangle}+c_{1} \pi^{\mu v} \nabla^{\alpha} u_{\alpha}+c_{2} \pi_{\alpha}^{\langle\mu} \sigma^{v\rangle \alpha}+c_{3} \pi_{\alpha}^{\langle\mu} \omega^{v\rangle \alpha}+c_{4} \pi_{\alpha}^{\langle\mu} \pi^{v\rangle \alpha} .
$$

Similar equations can be written for the other dissipative currents as well, in which case there are several new terms in the equations that couple the different dissipative currents. For more details, see e.g. Refs. [5, 6]. These equations together with the conservation laws are the basic equations governing the evolution of relativistic fluids. Thus, in the fluid dynamical limit the evolution can be solved in terms of $T^{\mu v}$ and the charge currents $N_{i}^{\mu}$ alone, provided that their initial values are given together with the transport coefficients and the equation of state.

The viscosity obviously modifies the spacetime evolution of macroscopic fields $T^{\mu v}$ and $N_{i}^{\mu}$, but these fields cannot be observed directly. What actually is observed, are the final momentum spectra of hadrons, and in fluid dynamical models of the heavy ion collisions it is necessary to know how the macroscopic field are translated into the local momentum distributions. This is usually done by using the so called 14 moment approximation, where for example the viscous corrections to the equilibrium distribution function $f_{0}$ due to shear viscosity are written as $\delta f \propto$ $f_{0} p_{\mu} p_{v} \pi^{\mu v}$, where $p^{\mu}$ is the 4-momentum of the particle. The momentum dependence of the 14 moment approximation is not unique, but it should be considered as one of the contributions to the full moment expansion [5]. Currently, the momentum dependence of $\delta f$ corrections is one of the major uncertainties in the fluid dynamical models, see e.g. Refs. [7, 8].

\section{Viscosity and flow coefficients in fluid dynamical models}

The most direct observable that limits the possible values of the transport coefficients is the azimuthal structure of the hadron transverse momentum spectra. The azimuthal part of the spectrum is usually characterized in terms of the flow coefficients $v_{n}$, defined through the Fourier expansion,

$$
v_{n} e^{i n \Psi_{n}}\left(p_{T}, y\right)=\left\langle e^{i n \phi}\right\rangle_{\phi} /\langle 1\rangle_{\phi},
$$

where the angular brackets $\langle\cdots\rangle_{\phi}$ denote the average $\int d \phi d N / d y d p_{T}^{2} d \phi(\cdots)$, and $\Psi_{n}$ is the eventplane angle that gives the direction of the deformation. In fluid dynamical models the $v_{n}$ depends on the magnitude of the initial spatial deformations, and on how effectively they are converted to the final momentum-space asymmetries during the evolution. This efficiency then depends on the properties of the matter.

On the average the "elliptic flow" coefficient $v_{2}$ is the largest of the $v_{n}$ coefficients, due to the average nuclear overlap geometry, and it gives the most direct constrain for the shear viscosity to entropy density ratio $\eta / s$. Several different model calculations $[9,10,11]$ suggest that this ratio has to be small in order to describe the data. For example in Ref. [11] a limit $1<4 \pi \eta / s<2.5$ was obtained, with the main uncertainty coming from the uncertainty in the initial conditions. A 
simultaneous description of all the $v_{n}$ coefficients give tighter constraints to the possible initial conditions, e.g. in Ref. [12] it was shown that dissipative fluid dynamics with the IP-Glasma initial conditions can reproduce the measured $v_{n}$ coefficients both at RHIC and the LHC energies. However, it was also found that description of the RHIC data requires smaller $\eta / s \sim 0.12$ than the LHC data that requires $\eta / s \sim 0.20$. A similar conclusion was obtained in Ref. [13]. In these estimates it was assumed that $\eta / s$ is a constant independent of temperature. In reality, $\eta / s$ can have a strong temperature dependence, and a constant $\eta / s$ should be thought as an effective $\eta / s$ integrated over the whole evolution history. In practice, by assuming that $\eta / s$ has a minimum around the QCD phase transition temperature, the constant $\eta / s$ estimates give the maximum value of minimum $\eta / s$.

At one collision energy it is possible to construct many different temperature dependencies of $\eta / s(T)$ that give an equally good description of the $v_{n}$ data $[14,15]$, but simultaneous description of the collision at different energies give more tighter constraints, as different temperature regions are weighted differently at different energies [16]. Moreover, besides the $v_{n}$ values themselves, the event-by-event correlations between $v_{n}$ s and their phases $\Psi_{n}$ give an additional independent constraint to initial conditions [17] and $\eta / s(T)$ [15]. A simultaneous analysis of $v_{n}$ s and their correlations at the top RHIC and LHC energies can be used to rule out several possible $\eta / s(T)$ parametrizations [15]. More constraints can be obtained from the lower energy collisions, and so far it has been demonstrated that a fluid dynamical model coupled to the UrQMD hadron cascade can also give a good description of the flow data [18] in these collision energies.

There is no reason to expect that bulk viscosity is negligible, especially near the QCD phase transition, and recently it was found [19] that a non-zero bulk viscosity can help to explain certain features of the data better. Particularly, because large bulk viscosity helps to reduce the average transverse momentum of the hadrons. Constraining bulk viscosity is also important in finding the limits for $\eta / s$, as non-zero bulk viscosity causes also similar suppression of the flow coefficients as the shear viscosity, see e.g. Ref. [20].

The details of the initial density profiles remain a significant background in the extraction of the transport coefficients, and it is essential that they are constrained simultaneously. The ratio $v_{2} / v_{3}$ [21], and the event-by-event $v_{2}$ fluctuations [22] provide such constraints that are more independent of the details of the fluid dynamical evolution. For example, both the $v_{2} / v_{3}$ ratio and the $v_{2}$ fluctuation spectra can be described simultaneously with the QCD based initial conditions IPGlasma [23] and EKRT model [15]. Moreover, the fluctuations of $v_{n}$ in the longitudinal direction are providing more constraints for the rapidity dependence of the initial conditions [24, 25].

\section{Small systems and applicability of fluid dynamics}

Recently, large flow coefficients have also been observed in much smaller systems, e.g. in proton-nucleus, and even in high-multiplicity proton-proton collisions. At least some of these measurements can be described fluid dynamical models [26, 27, 28, 29, 30, 31], but it is not yet clear whether fluid dynamics is truly applicable in these systems. It is not trivial to fulfill the condition of small Knudsen number even in heavy-ion collisions, and the situation is even less trivial in smaller collision systems [32, 33].

It has become evident that the large $v_{n}$ coefficients alone should not taken as the direct evidence of a fluid dynamical behavior, but e.g. parton cascades with perturbative QCD cross-sections can 
generate such signals $[34,35]$. One of the fundamental features of fluid dynamics is that the dynamics of the system is controlled by the equation of state and the transport coefficients alone, i.e. the rest of the microscopic details play no role. Thus, in the future, the important step in demonstrating fluid dynamical behavior (or lack of it) will be to demonstrate that all the different collisions systems and energies that are expected to show such behavior can be described by a single set of (temperature and chemical potential dependent) transport coefficients and equation of state, as those are the fundamental properties of thermalized matter and should not depend how the system was created, but only on the local state of the matter. It is also clear that such approach becomes computationally very expensive.

A practical way to perform such a global statistical analysis is through emulators, that essentially interpolates between the pre-calculated full model runs [36, 37, 38]. Through these approaches the computational cost can be reduced, and simultaneously they give a more systematic way to extract the properties of the matter itself.

\section{References}

[1] U. Heinz and R. Snellings, Ann. Rev. Nucl. Part. Sci. 63, 123 (2013) [arXiv:1301.2826 [nucl-th]]; P. Huovinen, Int. J. Mod. Phys. E 22, 1330029 (2013) [arXiv:1311.1849 [nucl-th]].

[2] W. A. Hiscock and L. Lindblom, Annals Phys. 151, 466 (1983).

[3] S. Pu, T. Koide and D. H. Rischke, Phys. Rev. D 81, 114039 (2010) [arXiv:0907.3906 [hep-ph]].

[4] W. Israel and J. M. Stewart, Annals Phys. 118, 341 (1979).

[5] G. S. Denicol, H. Niemi, E. Molnar and D. H. Rischke, Phys. Rev. D 85, 114047 (2012) [Phys. Rev. D 91, no. 3, 039902 (2015)] [arXiv:1202.4551 [nucl-th]].

[6] E. Molnár, H. Niemi, G. S. Denicol and D. H. Rischke, Phys. Rev. D 89, no. 7, 074010 (2014) [arXiv:1308.0785 [nucl-th]].

[7] K. Dusling, G. D. Moore and D. Teaney, Phys. Rev. C 81, 034907 (2010) [arXiv:0909.0754 [nucl-th]].

[8] D. Molnar and Z. Wolff, arXiv:1404.7850 [nucl-th].

[9] P. Romatschke and U. Romatschke, Phys. Rev. Lett. 99, 172301 (2007) [arXiv:0706.1522 [nucl-th]].

[10] M. Luzum and P. Romatschke, Phys. Rev. C 78, 034915 (2008) [Phys. Rev. C 79, 039903 (2009)] [arXiv:0804.4015 [nucl-th]].

[11] H. Song, S. A. Bass, U. Heinz, T. Hirano and C. Shen, Phys. Rev. Lett. 106, 192301 (2011) [Phys. Rev. Lett. 109, 139904 (2012)] [arXiv:1011.2783 [nucl-th]].

[12] B. Schenke, P. Tribedy and R. Venugopalan, Phys. Rev. Lett. 108, 252301 (2012) [arXiv:1202.6646 [nucl-th]].

[13] C. Shen, S. A. Bass, T. Hirano, P. Huovinen, Z. Qiu, H. Song and U. Heinz, J. Phys. G 38, 124045 (2011) [arXiv:1106.6350 [nucl-th]].

[14] R. Paatelainen, K. J. Eskola, H. Niemi and K. Tuominen, Phys. Lett. B 731, 126 (2014) [arXiv:1310.3105 [hep-ph]].

[15] H. Niemi, K. J. Eskola and R. Paatelainen, arXiv:1505.02677 [hep-ph]. 
[16] H. Niemi, G. S. Denicol, P. Huovinen, E. Molnar and D. H. Rischke, Phys. Rev. Lett. 106, 212302 (2011) [arXiv:1101.2442 [nucl-th]]; Phys. Rev. C 86, 014909 (2012) [arXiv:1203.2452 [nucl-th]].

[17] Z. Qiu and U. Heinz, Phys. Lett. B 717, 261 (2014) [arXiv:1208.1200 [nucl-th]].

[18] I. A. Karpenko, P. Huovinen, H. Petersen and M. Bleicher, Phys. Rev. C 91, no. 6, 064901 (2015) [arXiv:1502.01978 [nucl-th]].

[19] S. Ryu, J.-F. Paquet, C. Shen, G. S. Denicol, B. Schenke, S. Jeon and C. Gale, Phys. Rev. Lett. 115, no. 13, 132301 (2015) [arXiv:1502.01675 [nucl-th]].

[20] J. Noronha-Hostler, J. Noronha and F. Grassi, Phys. Rev. C 90, no. 3, 034907 (2014) [arXiv:1406.3333 [nucl-th]].

[21] E. Retinskaya, M. Luzum and J. Y. Ollitrault, Phys. Rev. C 89, no. 1, 014902 (2014) [arXiv:1311.5339 [nucl-th]].

[22] H. Niemi, G. S. Denicol, H. Holopainen and P. Huovinen, Phys. Rev. C 87, no. 5, 054901 (2013) [arXiv:1212.1008 [nucl-th]].

[23] B. Schenke and R. Venugopalan, Phys. Rev. Lett. 113, 102301 (2014) [arXiv:1405.3605 [nucl-th]].

[24] L. G. Pang, G. Y. Qin, V. Roy, X. N. Wang and G. L. Ma, Phys. Rev. C 91, no. 4, 044904 (2015) [arXiv:1410.8690 [nucl-th]].

[25] P. Bozek, W. Broniowski and A. Olszewski, Phys. Rev. C 91, 054912 (2015) [arXiv:1503.07425 [nucl-th]].

[26] K. Werner, M. Bleicher, B. Guiot, I. Karpenko and T. Pierog, Phys. Rev. Lett. 112, no. 23, 232301 (2014) [arXiv:1307.4379 [nucl-th]].

[27] J. L. Nagle et al., Phys. Rev. Lett. 113, no. 11, 112301 (2014) [arXiv:1312.4565 [nucl-th]].

[28] P. Bozek, W. Broniowski and G. Torrieri, Phys. Rev. Lett. 111, 172303 (2013) [arXiv:1307.5060 [nucl-th]].

[29] K. Werner, B. Guiot, I. Karpenko and T. Pierog, Phys. Rev. C 89, no. 6, 064903 (2014) [arXiv:1312.1233 [nucl-th]].

[30] I. Kozlov, M. Luzum, G. S. Denicol, S. Jeon and C. Gale, Nucl. Phys. A 931, 1045 (2014) [arXiv:1412.3147 [nucl-th]].

[31] A. Adare et al. [PHENIX Collaboration], Phys. Rev. Lett. 115, no. 14, 142301 (2015) [arXiv:1507.06273 [nucl-ex]].

[32] A. Bzdak, B. Schenke, P. Tribedy and R. Venugopalan, Phys. Rev. C 87, no. 6, 064906 (2013) [arXiv:1304.3403 [nucl-th]].

[33] H. Niemi and G. S. Denicol, arXiv:1404.7327 [nucl-th].

[34] J. Uphoff, F. Senzel, O. Fochler, C. Wesp, Z. Xu and C. Greiner, Phys. Rev. Lett. 114, no. 11, 112301 (2015) [arXiv:1401.1364 [hep-ph]].

[35] A. Bzdak and G. L. Ma, Phys. Rev. Lett. 113, no. 25, 252301 (2014) [arXiv:1406.2804 [hep-ph]].

[36] J. Novak, K. Novak, S. Pratt, J. Vredevoogd, C. Coleman-Smith and R. Wolpert, Phys. Rev. C 89, no. 3, 034917 (2014) [arXiv:1303.5769 [nucl-th]].

[37] J. E. Bernhard, P. W. Marcy, C. E. Coleman-Smith, S. Huzurbazar, R. L. Wolpert and S. A. Bass, Phys. Rev. C 91, no. 5, 054910 (2015) [arXiv:1502.00339 [nucl-th]].

[38] S. Pratt, E. Sangaline, P. Sorensen and H. Wang, Phys. Rev. Lett. 114, 202301 (2015) [arXiv:1501.04042 [nucl-th]]. 